\title{
Heliospheric tracking of enhanced density structures of the 6 October 2010 CME
}

\author{
Wageesh Mishra ${ }^{1, *}$ and Nandita Srivastava ${ }^{1,2}$ \\ 1 Udaipur Solar Observatory, Physical Research Laboratory, Udaipur 313001, India \\ ${ }^{*}$ Corresponding author: wageesh@prl.res.in \\ 2 Center of Excellence in Space Sciences India (CESSI), IISER Kolkata, Mohanpur 741246, India
}

Received 21 November 2014 / Accepted 13 June 2015

\begin{abstract}
A Coronal Mass Ejection (CME) is an inhomogeneous structure consisting of different features which evolve differently with the propagation of the CME. Simultaneous heliospheric tracking of different observed features of a CME can improve our understanding about relative forces acting on them. It also helps to estimate accurately their arrival times at the Earth and identify them in in-situ data. This also enables finding any association between remotely observed features and in-situ observations near the Earth. In this paper, we attempt to continuously track two density enhanced features in the CME of 6 October 2010, one at the front and another at the rear edge. This is achieved by using time-elongation maps constructed from STEREO/SECCHI observations. We derive the kinematics of the tracked features using various reconstruction methods. The estimated kinematics are used as inputs in the Drag Based Model (DBM) to estimate the arrival time of the tracked features of the CME at L1. On comparing the estimated kinematics as well as the arrival times of the remotely observed features with in-situ observations by Advanced Composition Explorer $(A C E)$ and Wind, we find that the tracked bright feature at the rear edge of 6 October 2010 CME corresponds most probably to the enhanced density structure after the magnetic cloud detected by $A C E$ and Wind. In-situ plasma and compositional parameters provide evidence that the rear edge density structure may correspond to a filament associated with the CME while the density enhancement at the front corresponds to the leading edge of the CME. Based on this single event study, we discuss the relevance and significance of Heliospheric Imager (HI) observations in identification of the three-part structure of the CME.
\end{abstract}

Key words. Corona - Sun - Heliosphere

\section{Introduction}

A CME is huge magnetized plasma eruption from the Sun into the heliosphere and can be observed due to Thomson scattering of photospheric light off free electrons in the corona (Billings 1966; Howard \& Tappin 2009). In coronagraphic observations, a classic CME displays the so-called three-part structure. This includes a leading edge, which is followed by a dark cavity and a bright core (Illing \& Hundhausen 1985). The Solar TErrestrial RElations Observatory (STEREO; Howard et al. 2008) Heliospheric Imager (HI; Eyles et al. 2009) era has proved to be a boon for a solar terrestrial physicist in tracking a CME. However, the identification of different features of CMEs in the heliosphere by their continuous tracking and the prediction of their arrival time at $1 \mathrm{AU}$ has been achieved with limited accuracy only (Liu et al. 2010a; Howard \& DeForest 2012; Liu et al. 2013; Mishra \& Srivastava 2013, 2014; Mishra et al. 2015). Due to lack of information regarding the evolution of a CME during its propagation between the Sun and the Earth, and the process by which it manifests itself in ambient solar wind, sometimes, its association in in-situ observations is difficult. This is in part because of the challenges in extracting the faint Thomson scattered signal from the brighter background signals dominated by instrumental stray light, F-corona and background starfield (Eyles et al. 2009). The heliospheric tracking of CMEs has been successfully attempted using $J$-map, which is an elongation versus time plot at a certain position angle constructed from a sequence of stacked images (Sheeley et al. 1999; Davies et al. 2009). Such attempts have mostly dealt with tracking of a CME front and associated it with the sheath observed prior to the leading edge in in-situ data (Davis et al. 2009; Liu et al. 2010a, 2011; Möstl et al. 2011; Mishra \& Srivastava 2013). In a rare attempt, Howard \& DeForest (2012) tracked a cavity like feature (in coronagraph images) of a classical CME using $\mathrm{HI}$ images which could be associated with a magnetic cloud identified in in-situ data near the Earth. Also, DeForest et al. (2011) have attempted to identify different CME structures in STEREO/HI2 observations and compared them with in-situ features detected near $1 \mathrm{AU}$. Sometimes, the observed features in coronagraphic images become too faint to be detected in $\mathrm{HI}$ images and to be tracked in $J$-maps, especially for an Earthdirected feature when STEREO is behind the Sun. In the absence of continuous tracking of a CME from the Sun to the Earth, the extrapolation of obtained heliospheric kinematics out to the Earth or use of these kinematics in appropriate models (e.g. drag based model) is done currently to predict the arrival time of CME at L1 (Liu et al. 2010a; Colaninno et al. 2013; Mishra \& Srivastava 2013).

Over the last decade, with the availability of heliospheric imaging observations, a number of reconstruction methods have been developed by various authors (Tappin et al. 2004; Howard et al. 2006; Kahler \& Webb 2007; 
Sheeley et al. 2008; Lugaz et al. 2009, 2010; Liu et al. 2010a; Davies et al. 2012, 2013). These methods are based on a number of assumptions on the geometry and evolution of CMEs. Therefore, prediction of CME arrival time, even if tracked continuously out to larger elongations, is not very accurate (Davis et al. 2010; Lugaz 2010; Mishra et al. 2014). The reconstruction methods enable the estimation of kinematics using the elongation variations of solar wind transients like CMEs. Therefore, the accuracy in remote identification, tracking and extraction of elongation angles of various CME features is of prime concern for their association with in-situ data and for the estimation of accurate arrival time.

A CME shows large-scale inhomogeneous structures in terms of density and magnetic field. Therefore, it is likely that these structures will be acted upon by unequal forces which can result in different kinematics. Occasionally, if a CME feature is missed by an in-situ spacecraft, the sequential tracking of other structures might be helpful in relating the remote observations and in-situ. Such a study is of twofold importance. Scientifically, the understanding of the physical nature of various features (leading edge, cavity and core) of a CME can help in the theoretical modelling of a CME to investigate its heliospheric evolution. On the other hand, different features of a CME may lead to different perturbations in the Earth's magnetosphere because of their dissimilar plasma and magnetic field parameters, which also need to be understood.

In this paper, we report on the evolution of the front and the rear edge of a filament associated geo-effective CME of the 6 October 2010. The layout of paper is as follows: in Section 2 we present the observations of the CME. In Section 3, we describe the methodology followed for the analysis of tracking of different features of the CME in COR and HI images. We discuss the results in Section 4.

\section{CME of 6 October 2010: observations and analysis approach}

We use the white light observations of the CME from the twin spacecraft of the STEREO mission and in-situ plasma and magnetic field parameters of the solar wind from the Advanced Composition Explorer (ACE; Stone et al. 1998) and Wind (Ogilvie et al. 1995) spacecraft. STEREO-A and STEREO-B move in such a way that the separation between them increases by about $45^{\circ}$ per year. Each STEREO spacecraft carries an identical imaging suite, called the Sun-Earth Connection Coronal and Heliospheric Investigation (SECCHI; Howard et al. 2008). SECCHI consists of five telescopes, namely, an EUV imager, two coronagraphs (COR1 and COR2) and two heliospheric imagers (HI1 and HI2). Combining the fields of view of both coronagraphs and both heliospheric imagers together, a $\mathrm{CME}$ can be imaged from elongation $0.4^{\circ}$ to $88.7^{\circ}$, i.e. from its birth in the inner corona all the way to the Earth and beyond. The $A C E$ and Wind spacecraft are located at the first Lagrangian point (L1) and measure the plasma and magnetic field parameters of the solar wind. At the launch time of the CME on 6 October 2010, the separation between the twin STEREO spacecraft was about $161^{\circ}$. The CME was associated with a prominence eruption located in the North-East (NE) quadrant of the solar disc as seen from the Earth's perspective. Figure 1 shows the filament eruption in the $304 \AA$ data from the Solar Dynamic Observatory/ Atmospheric Imaging Assembly (SDO/AIA; Lemen et al. 2012).

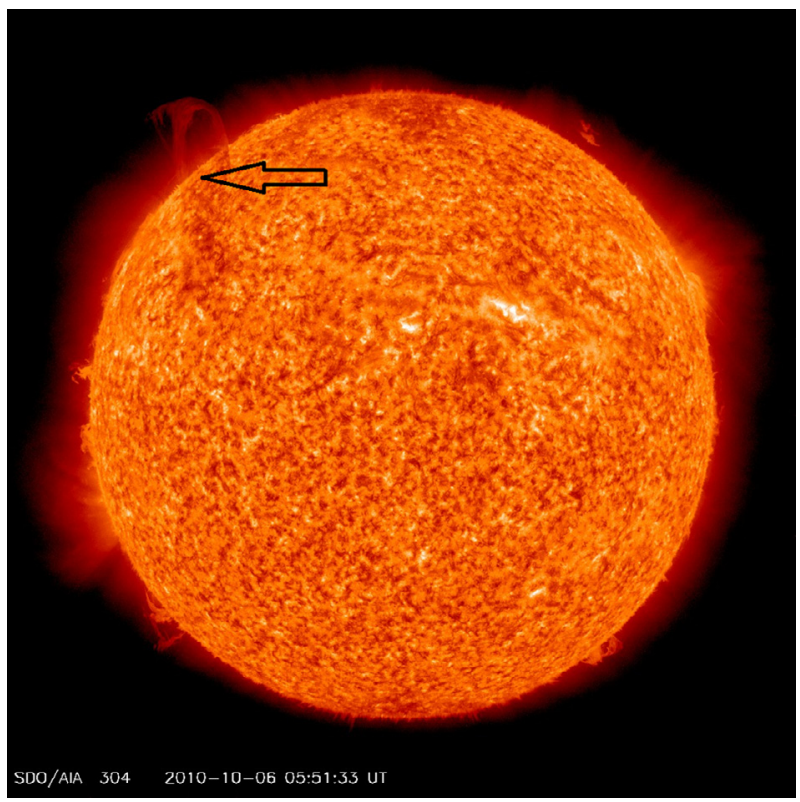

Fig. 1. The erupting filament material, shown with an arrow, on the NE quadrant of the Sun, observed in $304 \AA$ image from SDO/AIA.

The evolution of the 6 October $2010 \mathrm{CME}$ in COR and $\mathrm{HI}$ field of view is shown in Figure 2. The front/leading edge (termed F1) and core/associated filament (termed F2) could be easily identified in COR 1 and COR2 images. These features are marked with arrows (in black) in the top panels of Figure 2. By manual selection of the features F1 and F2 in both sets of twin STEREO images and application of the tie-pointing method of $3 \mathrm{D}$ reconstruction, their kinematics are estimated. As the CME approached the edge of the COR2 field of view, STEREO-A had a data gap until the CME appeared in the HI1-A field of view as a diffuse structure where its leading edge and core could not be distinguished as in the COR field of view. Therefore, we constructed the $J$-maps for this CME using running difference images of HI1 and HI2 data, and tracked the two density enhanced features at its front and rear edge. In the $J$-map the tracked features are termed Feature 1 and Feature 2 as we are not certain that these features are exactly the same as F1 and F2 tracked in the COR field of view. Feature 1 and Feature 2 are each marked with an arrow (in black) in the middle panel of Figure 2. Using derived elongation from $J$-maps for these features, we estimated their kinematics. Based on their arrival times, we attempted to identify them in in-situ observations and to associate them with the observed features in coronagraph observations.

Several 3D reconstruction methods have been developed in the STEREO era, to estimate the kinematics of CMEs based on different assumptions about geometry, speed and propagation direction. Some of the methods utilize Thomson scattering geometry and/or multiple-viewpoint observations. In a recent study, we have shown that different reconstruction methods (applied to HI observations) estimate different kinematic profiles of a CME (Mishra et al. 2014). We have also shown that stereoscopic methods which take into account the spherical geometry of a CME are superior to single-spacecraft reconstruction methods and single-spacecraft fitting methods. But to take into account the maximum uncertainties in our analysis, we use a total of 10 reconstruction methods. The use of 


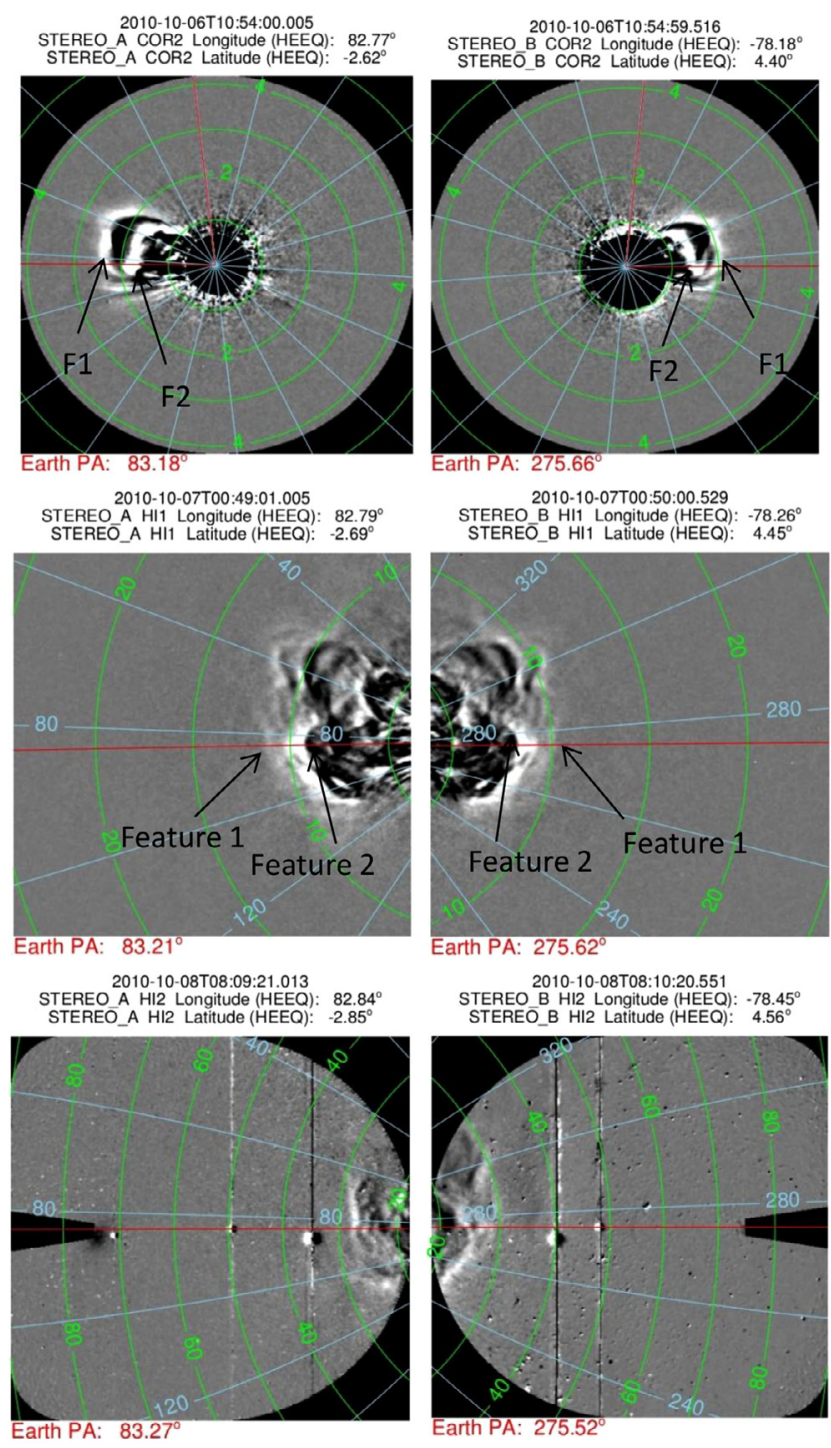

Fig. 2. Evolution of the 6 October $2010 \mathrm{CME}$ observed in COR2, HI1 and HI2 images from STEREO-A (left column) and STEREO-B (right column). Contours of elongation angle (green) and position angle (blue) are overplotted. The vertical red line in the COR 2 images marks the $0^{\circ}$ position angle contour. The horizontal lines (red) on all panels indicate the position angle of Earth. The features (F1 and F2) tracked in coronagraph fields of view and the features (Feature 1 and Feature 2) tracked in the HI field of view are shown with arrows (black) in the top and middle panels, respectively.

these methods also allows us to verify their performance by estimating the kinematic profiles and arrival time of tracked features. We have implemented seven single-spacecraft methods:

- Point-P (PP; Howard et al. 2006).

- Fixed-Phi (FP; Kahler \& Webb 2007).

- Harmonic Mean (HM; Lugaz et al. 2009).

- Self-Similar Expansion (SSE; Davies et al. 2012).

- Fixed-Phi Fitting (FPF; Rouillard et al. 2008).

- Harmonic Mean Fitting (HMF; Lugaz 2010).

- Self-Similar Expansion Fitting (SSEF; Davies et al. 2012).
These seven methods all require elongation measurements of a moving CME feature from a single viewpoint. We have also implemented three stereoscopic methods:

- Geometric Triangulation (GT; Liu et al. 2010b).

- Tangent to A Sphere (TAS; Lugaz et al. 2010).

- Stereoscopic Self-Similar Expansion (SSSE; Davies et al. 2013).

These three methods require simultaneous elongation measurements from two viewpoints to estimate the kinematics of a tracked feature.

It is to be noted that in FP, GT and FPF reconstruction methods, a CME is treated as a point, while PP, HM, HMF, SSE, SSEF, TAS and SSSE reconstruction methods consider a CME as a large-scale structure. In FPF, HMF and SSEF methods, a CME is assumed to propagate with a constant speed along a fixed direction. Therefore, different assumptions make these methods independent of each other to some extent (Mishra et al. 2014).

We used the estimated kinematics as inputs in the Vršnak et al. (2013) drag based model (DBM), for the distance over which the CME could not be tracked unambiguously in $J$-maps, and estimated the CME's arrival time at L1. The DBM model is based on the assumption that after a distance of $20 R_{\odot}$, the dynamics of a CME is governed completely by drag forces (Cargill et al. 1996; Cargill 2004) acting on it, and the drag acceleration is proportional to the square of the difference in speed of the CME and ambient solar wind. The proportionality constant is called the drag parameter, which lies in the range from $0.2 \times 10^{-7}$ to $2.0 \times 10^{-7} \mathrm{~km}^{-1}$ as per their analysis. We used these values of the drag parameter and chose the speed of the ambient solar wind medium as $350 \mathrm{~km} \mathrm{~s}^{-1}$, in agreement with the prevailing slow ambient solar wind speed for this CME. The obtained kinematics and arrival times of both tracked features are used to associate them with density enhanced structures observed in in-situ observations. We also attempt to find an association between the three-part structure of the CME seen in the COR field of view with features observed in HI and in in-situ data.

\section{Heliospheric tracking of the 6 October 2010 CME features}

\subsection{Remote-sensing observations}

In both sets of SECCHI/COR images, the front (leading edge) and filament (rear edge) of the 6 October 2010 CME are identified clearly. To estimate the $3 \mathrm{D}$ dynamics, we applied the tie-pointing method of $3 \mathrm{D}$ reconstruction by using the scc_measure.pro routine of the SolarSoft SECCHI library (Thompson 2009) to the feature on the leading edge (F1) and in the filament/core (F2) of the CME observed in COR1 and COR2 images. Prior to the implementation of the 3D reconstruction technique, coronagraphic images were processed following the procedure as described by Mierla et al. (2009). The data from the remote-sensing instruments on STEREO is taken from UKSSDC. ${ }^{1}$ The estimated 3D location, in the Stonyhurst heliographic coordinate system, for tracked

\footnotetext{
${ }^{1}$ UK Solar System Data Centre (http://www.ukssdc.ac.uk/solar/ stereo/data.html).
} 


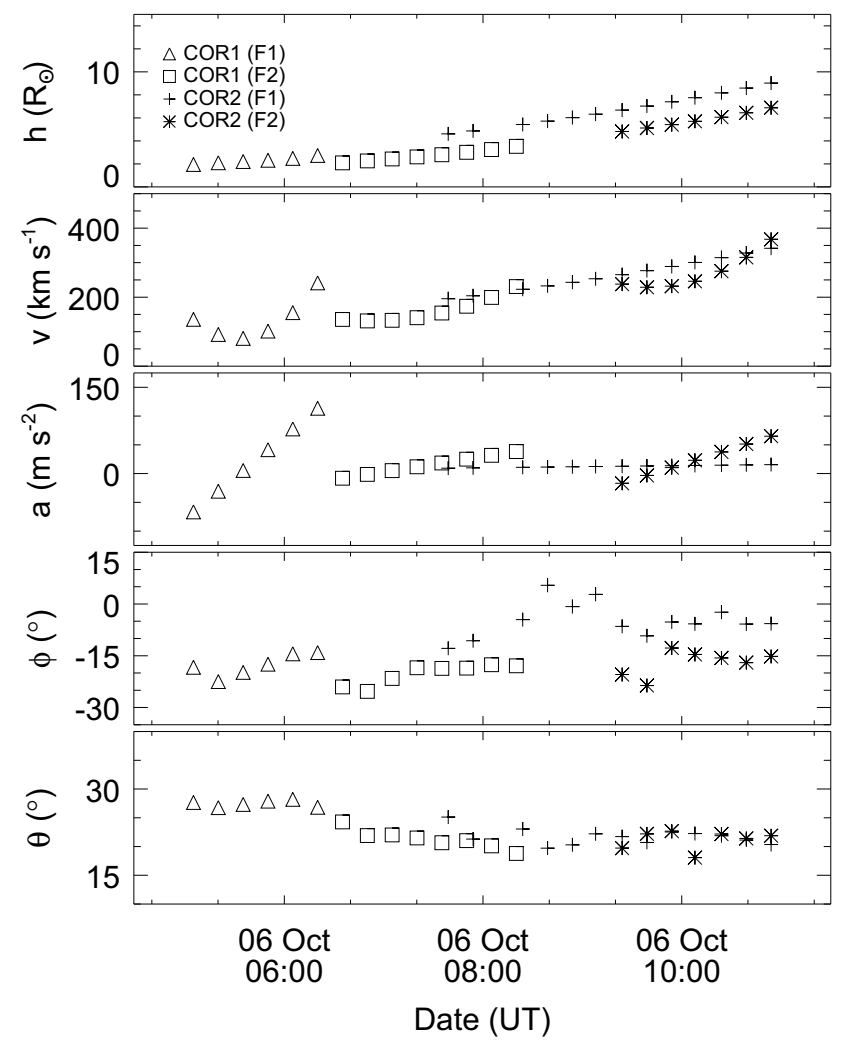

Fig. 3. Top to bottom panels show the evolution of height, speed, acceleration, longitude and latitude. Here F1 and F2 correspond to features on the leading edge and core/filament of the 6 October $2010 \mathrm{CME}$

features $(\mathrm{F} 1 \& \mathrm{~F} 2)$ is plotted in Figure 3 . We admit the possibility of human error in the identification of features in the pair of STEREO images on which tie-pointing method is performed. An earlier study by Joshi \& Srivastava (2011) has shown that an error of three pixels leads to errors of $0.12 R_{\odot}$ and $0.6 R_{\odot}$ in the distance estimates in the COR1 and COR2 fields of view, respectively, while the errors in longitude and latitude are less than $2^{\circ}$. Such negligibly small errors have no crucial implications on the results obtained in the present study.

From Figure 3, it is clear that both F1 and F2 move at around $30^{\circ}-20^{\circ}$ north from the ecliptic in the coronagraphic field of view and are eastward of the Sun-Earth line. The estimated kinematics show that they follow approximately the same trajectory in 3D space. The leading edge (F1) shows acceleration in the COR1 field of view while filament (F2) seems to accelerate more in the COR2 field of view than in the COR1 field of view. The leading edge (F1) becomes too diffuse to be tracked at the time when filament (F2) reached the COR1 field of view. From Figure 3, it is clear that the separation between the features $\mathrm{F} 1$ and $\mathrm{F} 2$ is approximately $1.0 R_{\odot}$ in the COR1 field of view. F1 and F2 could also be tracked in the COR2 field of view up to 10:54 UT on 6 October 2010. Because of a data gap from 10:54 UT until 17:39 UT in science images from STEREO-A, these features could not be tracked and reconstructed further. At the outermost tracked points in the COR2 field of view, the separation between F1 and F2 increased to $3 R_{\odot}$.

To track the CME features in the HI field of view, we used the $J$-map technique (Rouillard et al. 2008; Sheeley et al. 2008; Davies et al. 2009; Harrison et al. 2012) based on a method originally developed by Sheeley et al. (1999) to track features in coronagraph fields of view. We constructed the $J$-maps using running difference images of HI1 and HI2. The detailed procedure is explained in Mishra \& Srivastava (2013). Figure 4 shows $J$-maps where two positively inclined bright tracks which correspond to outward motion of two density structures can be seen. These bright tracks (marked with red and blue) could be due to features of two different CMEs or different features (front and rear edge) of the same CME passing along the ecliptic. We examined the background subtracted movies from COR and HI, COR1 CME catalogue (http://cor1.gsfc. nasa.gov/catalog/) and the LASCO CDAW catalogue (http:// cdaw.gsfc.nasa.gov/CME_list/), and found that no Earthdirected CME was launched close to the occurrence of the
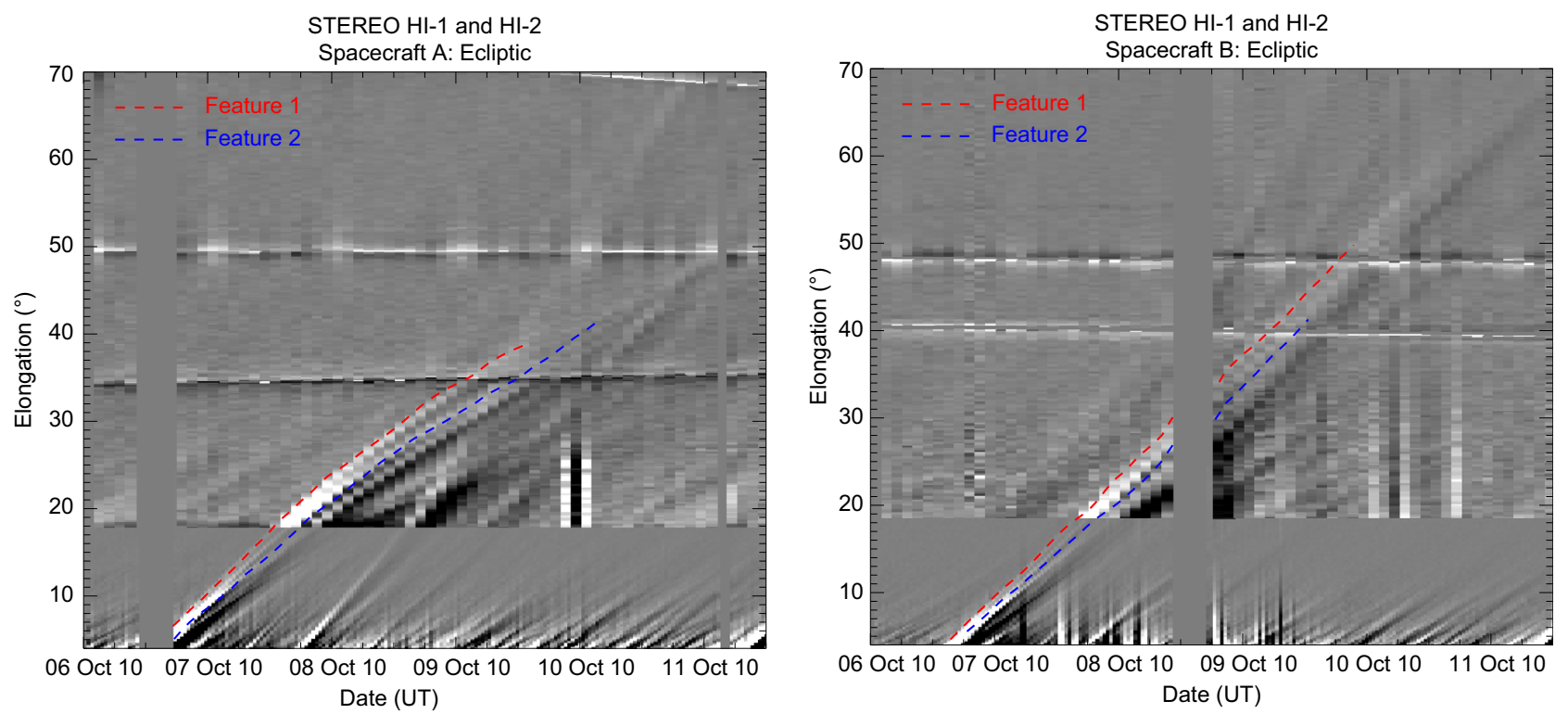

Fig. 4. Time-elongation maps ( $J$-maps) for STEREO-A (left) and STEREO-B (right) constructed from running differences images from HI 1 and HI2, for the time interval from 06 October 2010 to 11 October 12:00 UT, 2010. Two features (marked as Feature 1 and Feature 2, with red and blue lines, respectively) are tracked corresponding to enhanced density features. 
6 October 2010 CME. Therefore, we assume that these tracked features are two different features of the Earth-directed geoeffective CME of 6 October 2010. The presence of the planets Venus and Earth at elongations of $34.3^{\circ}$ and $49.5^{\circ}$, respectively, in the HI2-A field of view caused the two horizontal lines in the left panel of Figure 4. In the right panel of this figure, two horizontal lines are due to presence of these planets at elongations of $40.4^{\circ}$ and $48.2^{\circ}$ in the HI2-B field of view. A slanted line that appears in the left panel of Figure 4 on 10 October is due to the entrance of Jupiter into the HI2-A field of view.

We derived the elongation variation of both moving features (Feature 1 and Feature 2) by tracking the density enhancements that appear as positively inclined bright tracks in the $J$-maps (Fig. 4). These elongation angles are converted to distance using various reconstruction methods described in Section 2. Feature 1 and Feature 2 could be tracked out to $39^{\circ}$ and $41^{\circ}$ elongation, respectively, in STEREO- $A \mathrm{~J}$-maps. In STEREO-B J-maps, Features 1 and 2 could be tracked out to $50^{\circ}$ and $41^{\circ}$, respectively. In our earlier paper (Mishra et al. 2014), the analysis of the front edge (Feature 1) of the 6 October $2010 \mathrm{CME}$ is carried out by estimating its kinematics using all 10 methods (PP, FP, FPF, HM, HMF, SSE, SSEF, GT, TAS, SSSE) and their results are compared in detail. The obtained kinematics are used as inputs to the DBM to estimate the arrival time (for details, see Figs. 3-5 and Table 1 in Mishra et al. 2014). In the current paper, we focus on the analysis of the second feature (Feature 2).

We used the single-spacecraft PP, FP, HM and SSE methods to derive the kinematics of the tracked Feature 2. The FP, HM and SSE methods require the direction of propagation (longitude) of the tracked feature, which is obtained from the longitude (Fig. 3) estimated using the tie-pointing reconstruction method in the COR2 field of view. We fixed the propagation direction of this feature as $10^{\circ}$ East of the Sun-Earth line, which corresponds to a longitude difference of $93^{\circ}$ and $68^{\circ}$ from STEREO-A and $B$, respectively. In SSE method, we need to fix the angular half-width $(\lambda)$ of the CME (Davies et al. 2012) which is taken as $30^{\circ}$ in our case. The parameter $\lambda$ is related to the curvature of the CME front and hence it measures the width of the CME. The kinematics profile of tracked Feature 2 from STEREO-A and STEREO-B viewpoints is estimated and shown in Figures 5 and 6, respectively. The estimated kinematics over the outermost tracked points have been used in the DBM corresponding to extreme range of drag parameter to obtain its arrival time at L1 (Table 1). We also applied the three fitting methods (FPF, HMF and SSEF) to obtain a set of speed, propagation direction and launch time parameters that best reproduce the observed elongation-time profiles (extracted from $S T E R E O-A$ and $B J$-maps) of Feature 2. We took the angular half-width of the $\mathrm{CME}$ to be $30^{\circ}$ when applying the SSEF method. The retrieved best-fit parameters are used to estimate the arrival time of Feature 2 at L1 and are given in Table 1.

For associating remotely observed features with in-situ observations, we must correctly ascertain their direction of propagation in the heliosphere. The direction of propagation of tracked Feature 1 has been estimated using all the ten methods and was found to be towards the Earth (Mishra et al. 2014). If Feature 2 is not following the direction of propagation of Feature 1, then the same elongation for both the features will correspond to a different distance from the Sun. Therefore, we must examine the continuous evolution of

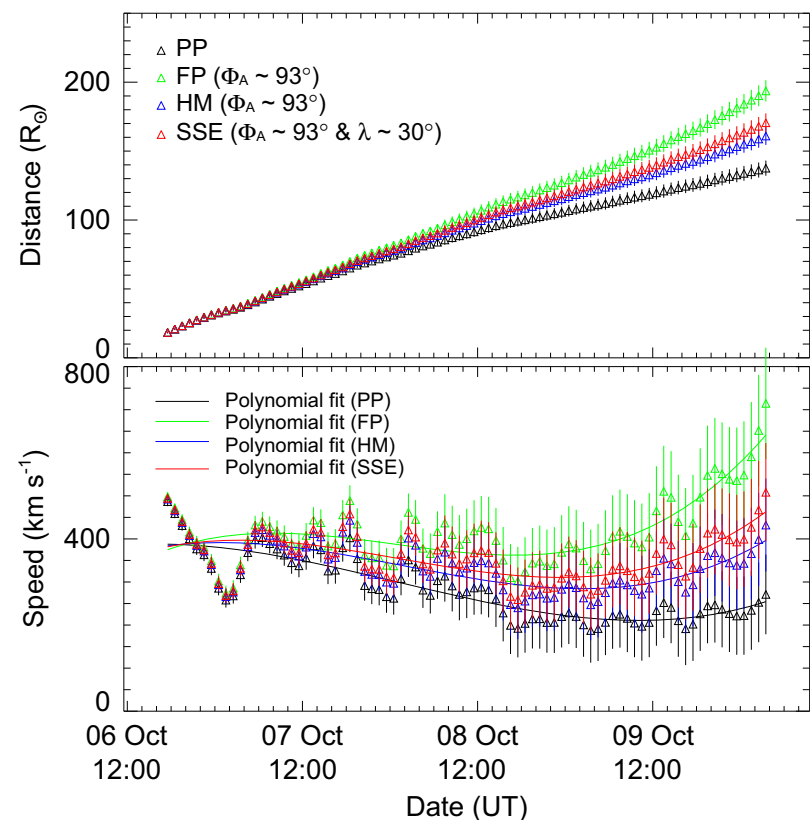

Fig. 5. Top panel shows the estimated distance profiles of Feature 2, based on application of the PP, FP, HM and SSE methods based on elongation-time variations estimated from STEREO-A J-maps. In the bottom panel, speed profiles derived from the adjacent distances using 3-point Lagrange interpolation (solid line shows the polynomial fit) are shown. Vertical lines show the error bars, derived by taking uncertainties of $3 \%$ and $4 \%$ in the estimated distance in the HI1 and HI2 fields of view, respectively.

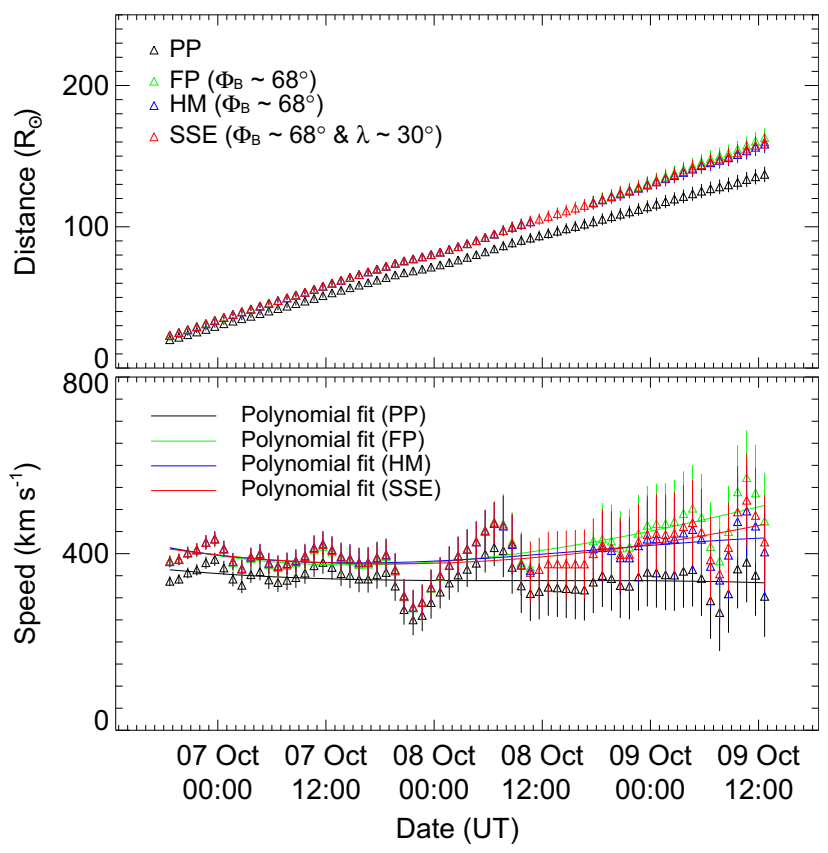

Fig. 6. As Figure 5, corresponding to application of methods on elongation-time variations estimated from STEREO-B J-maps.

Feature 2, the direction of propagation of which cannot be determined using single-spacecraft methods (e.g. PP, FP, HM, SSE, FPF, HMF, SSEF). For this, we also implemented the stereoscopic reconstruction methods, i.e. GT, TAS and SSSE 
Table 1. The kinematics input to the DBM and the resulting predicted arrival times and speeds (and errors therein) of Feature 2 at L1, corresponding to the extreme range of drag parameter. In the bottom panel, best-fit parameters, predicted arrival times and speeds (and errors therein) estimated from time-elongation track fitting methods are given. The STEREO-A and $B$ shown in parentheses for each method denotes the spacecraft from which data is used. Negative (positive) errors in predicted arrival time correspond to a predicted arrival time that is before (after) the actual CME arrival time determined from in-situ measurements. Negative (positive) errors in predicted speed correspond to a predicted speed that is less that (more than) than actual CME speed at L1.

\begin{tabular}{|c|c|c|c|c|c|}
\hline Methods & $\begin{array}{c}\text { Kinematics as } \\
\text { inputs in DBM } \\
\left(t_{0}, R_{0}\left[R_{\odot}\right]\right. \\
\left.v_{0}\left[\mathrm{~km} \mathrm{~s}^{-1}\right]\right)\end{array}$ & $\begin{array}{l}\text { Predicted arrival time } \\
\text { using kinematics }+ \\
\text { DBM (UT) }(\gamma=0.2 \\
\left.\text { to } 2.0\left[10^{-7} \mathrm{~km}^{-1}\right]\right)\end{array}$ & $\begin{array}{c}\text { Predicted transit } \\
\text { speed at L1 }\left(\mathrm{km} \mathrm{s}^{-1}\right) \\
(\gamma=0.2 \text { to } \\
\left.2.0\left[10^{-7} \mathrm{~km}^{-1}\right]\right)\end{array}$ & $\begin{array}{l}\text { Error in predicted } \\
\text { arrival time (hrs) } \\
(\gamma=0.2 \text { to } \\
\left.2.0\left[10^{-7} \mathrm{~km}^{-1}\right]\right)\end{array}$ & $\begin{array}{l}\text { Error in predicted } \\
\text { transit speed } \\
\left(\mathrm{km} \mathrm{s}^{-1}\right)(\gamma=0.2 \text { to } \\
\left.2.0\left[10^{-7} \mathrm{~km}^{-1}\right]\right)\end{array}$ \\
\hline PP $(S T E R E O-A)$ & $\begin{array}{c}10 \text { Oct. } 03: 30 \\
137,230\end{array}$ & $\begin{array}{l}12 \text { Oct. } 12: 51 \text { to } \\
12 \text { Oct. } 03: 22\end{array}$ & 270 to 327 & 23.7 to 14.2 & -85 to -28 \\
\hline $\mathrm{PP}(S T E R E O-B)$ & $\begin{array}{c}09 \text { Oct. } 12: 40 \text {, } \\
137,360\end{array}$ & $\begin{array}{l}11 \text { Oct. } 05: 14 \text { to } \\
11 \text { Oct. } 05: 21\end{array}$ & 360 to 358 & -8 to -7.8 & 5 to 3 \\
\hline $\mathrm{FP}(S T E R E O-A)$ & $\begin{array}{c}09 \text { Oct. 10:30, } \\
149,380\end{array}$ & $\begin{array}{l}10 \text { Oct. } 18: 54 \text { to } \\
10 \text { Oct. } 19: 27\end{array}$ & 378 to 368 & -18.2 to -17.6 & 23 to 13 \\
\hline $\mathrm{FP}(S T E R E O-B)$ & $\begin{array}{c}08 \text { Oct. } 23: 39, \\
130,464\end{array}$ & $\begin{array}{l}10 \text { Oct. } 11: 05 \text { to } \\
10 \text { Oct. } 15: 28\end{array}$ & 438 to 377 & -26.3 to -21.7 & 83 to 22 \\
\hline HM (STEREO-A) & $\begin{array}{c}09 \text { Oct. 11:30, } \\
132,285\end{array}$ & $\begin{array}{l}11 \text { Oct. } 16: 48 \text { to } \\
11 \text { Oct. } 12: 43\end{array}$ & 298 to 330 & 3.6 to -0.4 & -57 to -25 \\
\hline HM $(S T E R E O-B)$ & $\begin{array}{c}09 \text { Oct. } 12: 40, \\
158,430\end{array}$ & $\begin{array}{l}10 \text { Oct. } 13: 29 \text { to } \\
10 \text { Oct. } 15: 07\end{array}$ & 420 to 382 & -23.7 to -22.1 & 65 to 27 \\
\hline GT & $\begin{array}{l}09 \text { Oct. } 12: 40 \text {, } \\
159,450\end{array}$ & $\begin{array}{l}10 \text { Oct. } 12: 03 \text { to } \\
10 \text { Oct. } 14: 06\end{array}$ & 436 to 385 & -25.2 to -23.2 & 81 to 30 \\
\hline TAS & $\begin{array}{c}09 \text { Oct. } 12: 40 \text {, } \\
131,280\end{array}$ & $\begin{array}{l}11 \text { Oct. } 19: 21 \text { to } \\
11 \text { Oct. } 14: 41\end{array}$ & 295 to 330 & 6.1 to 1.4 & -60 to -25 \\
\hline SSSE & $\begin{array}{c}09 \text { Oct. } 12: 40, \\
154,385\end{array}$ & $\begin{array}{c}10 \text { Oct. } 18: 09 \text { to } \\
10 \text { Oct. } 18: 45\end{array}$ & 383 to 370 & -19.1 to -18.5 & 28 to 15 \\
\hline \multicolumn{6}{|c|}{ Time-elongation track fitting methods } \\
\hline Methods & $\begin{array}{l}\text { Best-fit parameters } \\
\left(t_{\alpha=0}, \Phi\left[^{\circ}\right]\right. \\
\left.v\left[\mathrm{~km} \mathrm{~s}^{-1}\right]\right) \\
\end{array}$ & $\begin{array}{l}\text { Predicted arrival } \\
\text { time at L1 (UT) }\end{array}$ & $\begin{array}{c}\text { Error in } \\
\text { predicted arrival } \\
\text { time } \\
\end{array}$ & $\begin{array}{c}\text { Error in } \\
\text { predicted speed at } \\
\mathrm{L} 1\left(\mathrm{~km} \mathrm{~s}^{-1}\right)\end{array}$ & Longitude $\left({ }^{\circ}\right)$ \\
\hline FPF $(S T E R E O-A)$ & $\begin{array}{l}06 \text { Oct. } 07: 44 \text {, } \\
90,378\end{array}$ & 10 Oct. $20: 29$ & -16.9 & 23 & -7 \\
\hline $\operatorname{FPF}(S T E R E O-B)$ & $\begin{array}{c}06 \text { Oct. } 04: 14 \text {, } \\
56.2,369\end{array}$ & 10 Oct. $19: 25$ & -17.7 & 14 & -21.5 \\
\hline HMF $(S T E R E O-A)$ & $\begin{array}{c}06 \text { Oct. 08:54, } \\
125,467\end{array}$ & 11 Oct. $07: 48$ & -5.4 & -18 & -42 \\
\hline HMF (STEREO-B) & $\begin{array}{c}06 \text { Oct. } 05: 51 \text {, } \\
58,378\end{array}$ & 11 Oct. $01: 35$ & -11.6 & -10 & -19.8 \\
\hline $\operatorname{SSEF}(S T E R E O-A)$ & $\begin{array}{c}06 \text { Oct. } 08: 40, \\
114,436\end{array}$ & 11 Oct. 09:02 & -4.2 & -13 & -31 \\
\hline SSEF $(S T E R E O-B)$ & $\begin{array}{c}06 \text { Oct. } 05: 37 \text {, } \\
57.4,377\end{array}$ & 11 Oct. $05: 43$ & -7.5 & -12 & -20.4 \\
\hline
\end{tabular}

(with $\lambda=30^{\circ}$ ), to estimate the kinematics of Feature 2 in the heliosphere. The GT and TAS methods are the triangulation version of the FP and HM methods. The GT and TAS methods assume extreme CME geometries while in the SSSE method the spherical structure for a CME can be fixed to an arbitrary size. Davies et al. (2013) have shown that the TAS and GT methods are special cases of the SSSE method. The details of these methods and their application to Feature 1 are discussed in Mishra et al. (2014). The obtained kinematics of Feature 2 using the GT, TAS and SSSE methods are presented in Figure 7. In this figure, the kinematics at the sunward edge of the HI1 field of view is not shown due to the occurrence of a singularity, where small uncertainties in elongation measurements lead to larger errors in kinematics (for details see, Liu et al. 2010a; Davies et al. 2013; Mishra \& Srivastava 2013; Mishra et al. 2014). In the middle and bottom panels of this figure, the direction and speed estimates from the singlespacecraft fitting methods are also overplotted. These estimates from STEREO-A and STEREO-B observations are plotted with dashed and dashed-dotted lines, respectively. Red, blue and green colours correspond to the FPF, HMF and SSEF methods, respectively.

Further, we input the kinematics estimated over the outer points into the DBM with an ambient solar speed of $350 \mathrm{~km} \mathrm{~s}^{-1}$ and the extremes of the range of the drag parameter. The estimated arrival time, transit speed at L1 and errors therein are given in Table 1. From the fifth and sixth columns of the table, it is clear that the errors in the predicted arrival time and speed are less when the maximum range of drag parameter is used in the DBM, for all the methods except for the three fitting methods. This highlights that a following feature (like Feature 2) of a CME probably experiences a larger drag force during its propagation from the Sun to the Earth. From Table 1, we also notice that different fitting methods give different estimates of Feature 2's propagation direction. Therefore, in light of our earlier study in Mishra et al. (2014) which 
W. Mishra and N. Srivastava: Heliospheric tracking of density structures of CME

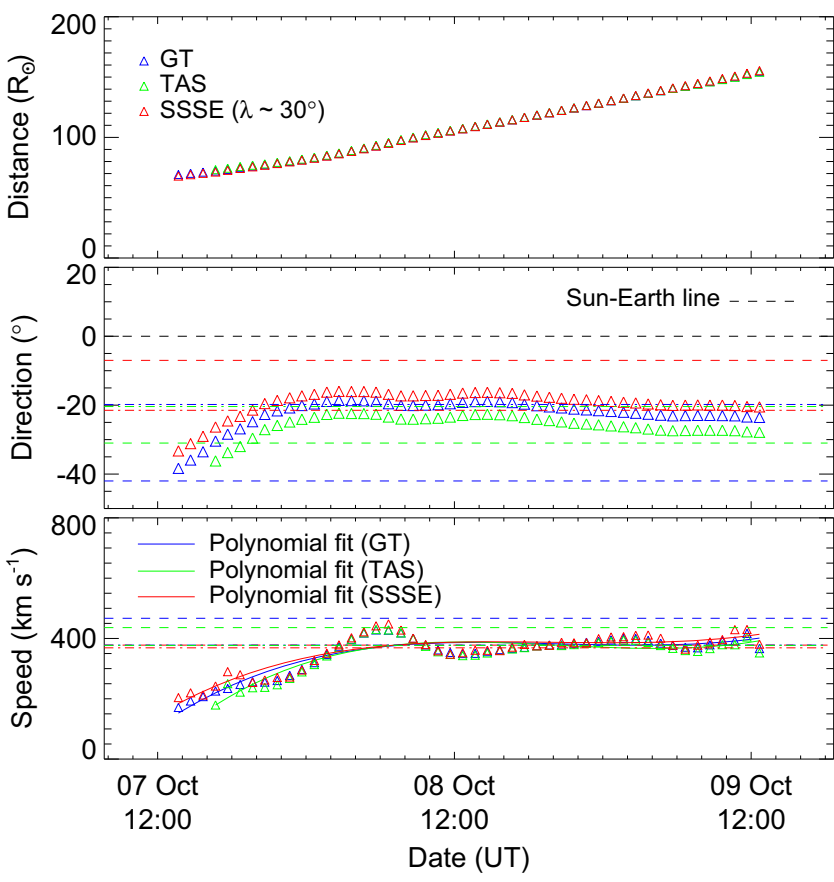

Fig. 7. From top to bottom, panels show the estimated distance, propagation direction and speed of the tracked Feature 2 using the GT, TAS and SSSE methods. In the middle and bottom panels, the direction and speed estimates from the single-spacecraft fitting methods are also overplotted. In these panels, dashed and dashed-dotted lines correspond to estimates from STEREO-A and STEREO-B observations, respectively. For these lines, the colors red, blue and green correspond to FPF, HMF and SSEF methods. In the middle panel, dashed horizontal line (black) marks the Sun-Earth line.

shows that stereoscopic methods provide reliable estimates of time variations of CME propagation direction, we rely on the CME direction estimated from GT, TAS and SSSE methods.

From Figures 5 and 6, we see that the PP and FP methods give the lowest and the highest estimates of distance of the tracked feature, respectively. The estimated distances from HM and SSE methods are intermediate between those obtained from PP and FP methods. In Figure 5, the observed unphysical late acceleration of Feature 2 is attributed to a possible deflection of the tracked feature far from the Sun. Although, the real deflection of features far from the Sun is rarely observed, this effect could result from it not being possible to track the same part of the CME leading edge in each successive image. This is especially true at large elongations where expansion of the overall CME plays an important role, leading to its artificial deflection (Howard 2011). Such effect will be more pronounced for the methods (e.g. FP, GT and FPF) in which the finite size of the CME is not taken into account. The performance of these single-spacecraft methods at larger elongations is sensitive to changes in the input values of propagation direction of CMEs used in the methods. Figures 5 and 6 also highlight that different values of CME propagation direction, used as inputs to the single-spacecraft methods, lead to different values of kinematic parameters. In our analysis, we have taken care that if, at any instant, the estimated kinematics become unreliable, due to breakdown of some assumptions made in the reconstruction methods, then the kinematics estimated prior to those points are used as inputs to the DBM. It must also be noted that if the position of the Earth or in-situ spacecraft at L1 is at different longitude than that of the ICME apex (the point of the CME leading edge at the largest heliospheric distance from the Sun), then a CME with a circular geometry (as modelled in HM and SSE methods) will lead to delay in the arrival times and lower estimated speeds at the Earth than that based on the CME apex kinematics. In this scenario, a CME can miss to hit the Earth. Therefore, values of speeds must be deprojected along the Sun-Earth line before extrapolating or using them in the DBM for arrival time prediction at L1. The direction of propagation of Feature 2, as estimated from the fitting methods (FPF, HMF and SSEF) is significantly away (up to $42^{\circ}$ east) from the Sun-Earth line, therefore an off-apex correction is applied on the speed obtained from these methods (Möstl et al. 2011; Möstl \& Davies 2013). In principle, such an off-axis correction should also be applied for HM, SSE, TAS, and SSSE methods. However the estimated direction of propagation of Feature 2 from these methods is within $\pm 25^{\circ}$ from the Sun-Earth line, hence such a correction would decrease the speed by only a few tens of $\mathrm{km} \mathrm{s}^{-1}$.

On comparing the two features, Feature 1 (Fig. 5 in Mishra et al. 2014) and Feature 2 (Fig. 7, this paper), we see that both moved approximately in the same direction through the heliosphere. Thus, both these Earth-directed features are likely to be detected by an in-situ spacecraft located at L1. Since, we have no information on the temperature, composition of plasma and magnetic field parameters associated with these tracked features; we can only rely on their estimated arrival time at L1 to relate features in in-situ data with Feature 1 and Feature 2. It is noted that while applying several reconstruction methods, we have considered the same angular width for the leading edge and core of the CME which may not be true. Moreover, we have assumed a convex geometry for both the leading edge and core of the CME. Although, we are aware that the convex geometry may not hold good for the core of the CME. This assumption may be invalid even for leading edge of the CME at larger elongations due to its possible flattening. However, in the absence of knowledge about the geometry and the angular width of the core and the leading edge of the CME, we have used the existing methods based on idealistic assumptions (Davies et al. 2013; Mishra et al. 2014), which is a pragmatic approach.

\subsection{Comparison of kinematics of tracked Features 1 and 2}

We tracked the two different features as the front (leading) and rear (trailing) edge of the CME in the STEREO/HI $J$-maps and derived their kinematics. The estimated separation between Feature 1 and Feature 2 based on their height derived from different reconstruction methods is shown in Figure 8. We found that the separation of the two features increased with time (Figs. 3 and 8) from $1.2 R_{\odot}$ to $15 R_{\odot}$ during their evolution from the COR to HI field of view. The estimated separation may be due to unequal driving forces on different features or due to a overall CME expansion or due to a combination of both. Figure 8 (the four panels from the bottom) shows the separation estimates by using single-spacecraft methods independently on STEREO-A and $B$ observations. It is evident that the separation increased from around $3 R_{\odot}$ to $11.3 R_{\odot}$ for PP, to $21.7 R_{\odot}$ for FP and to $15.4 R_{\odot}$ for HM. For the SSSE, TAS and GT techniques (the three top panels in Fig. 8), the separation between Feature 1 and Feature 2 increased out 


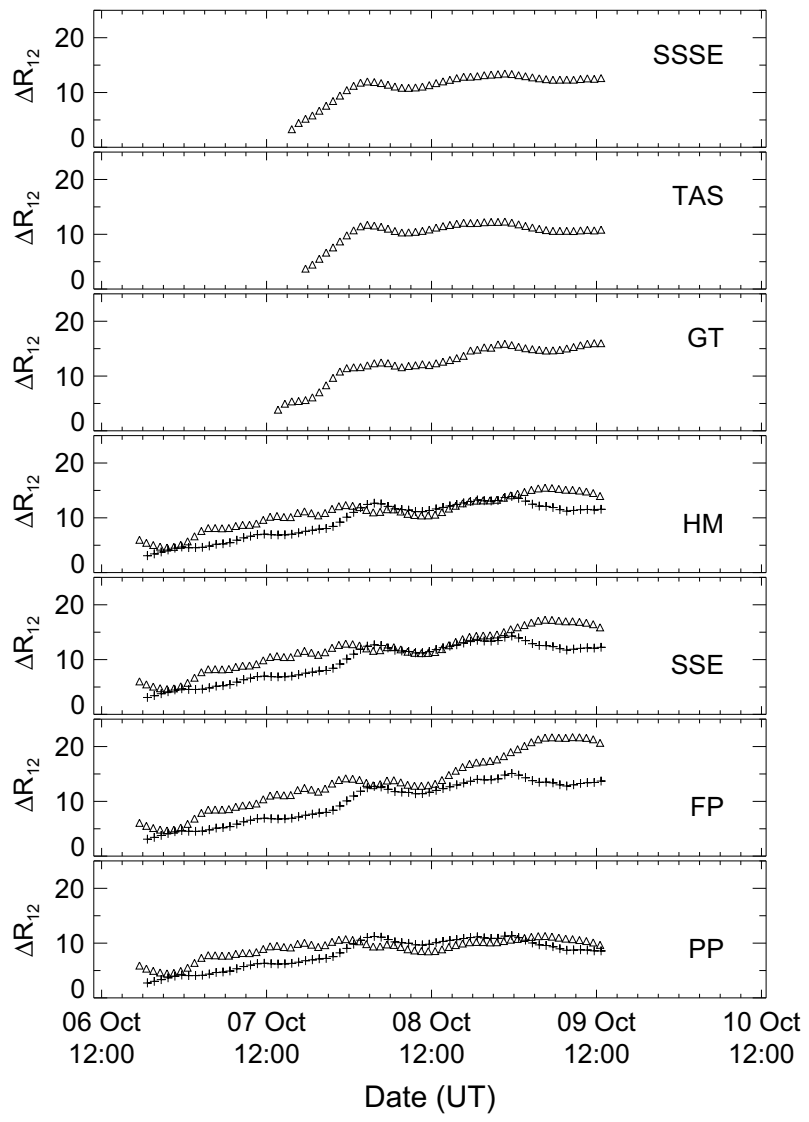

Fig. 8. Variation in separation distance (in unit of $R_{\odot}$ ) between the two tracked features with time. Each panel shows computed separation of the two features, based on the use of different methods for the estimation of distance of tracked Feature 1 and Feature 2. From top to bottom, panels correspond to SSSE, TAS, GT (twin spacecraft reconstruction), HM, SSE, FP and PP (single-spacecraft reconstruction) methods. In the four panels from bottom to top, the results derived by implementing the methods on STEREO-A and STEREO- $B$ observations are shown with triangles and pluses, respectively.

to $16.0,12.5$ and $13.5 R_{\odot}$, respectively, and became approximately constant at a distance of $\approx 120 R_{\odot}$.

Due to a singularity at the sunward edge of the HI1 field of view in GT, TAS and SSSE methods, the separation between the tracked features (Feature 1 and Feature 2) is estimated to be slightly smaller than the observed separation in the COR field of view. However, these initial erroneous estimates of separation can be avoided in the light of results obtained from GT, TAS and SSSE methods at larger elongations and estimates from other methods. Considering all the reconstruction methods applied to the SECCHI/HI observations, the estimated separation is probably somewhere between $11.2 R_{\odot}$ and $21.7 R_{\odot}$.

Several authors have shown that, at large distances from the Sun, the kinematic evolution of CMEs is mostly attributable to the drag force between the CMEs and the ambient solar wind (Cargill 2004; Manoharan 2006; Vršnak et al. 2010). In the present case, both of the features are tracked out to sufficiently large distances beyond the HI1 field of view where they attain speed close to that of the ambient solar wind, such that the driving forces on Feature 1 and Feature 2 become equal. The expansion speed of a CME at larger distance is more likely due to the high internal thermal pressure of the CME than the ambient solar wind pressure (Schwenn et al. 2005; Gopalswamy et al. 2009; Michalek et al. 2009; Poomvises et al. 2010). In our case, we notice that the expansion speed of the CME is $\approx 165 \mathrm{~km} \mathrm{~s}^{-1}$ at the entrance of the $\mathrm{HI}$ field of view which becomes negligibly small compared to radial speed of the $\mathrm{CME}$ at a distance of $115 R_{\odot}$. Therefore, under the aforementioned constraints regarding expansion and equal drag on both features of the $\mathrm{CME}$, it is expected that the final estimate of the separation between Feature 1 and Feature 2 will be approximately maintained out to the L 1 point. If a constant speed of $350 \mathrm{~km} \mathrm{~s}^{-1}$ is assumed for both features beyond the last observation points in the HI field of view, then the difference in their arrival time at L1 will range from 6.1 to $11.9 \mathrm{~h}$.

\subsection{Identification of remotely observed tracked features in near-Earth in-situ observations}

The formation and evolution of the three-part structure of a CME is not well understood. It is agreed that the leading edge, which appears bright, is due to the sweeping up of coronal plasma by erupting flux ropes or the presence of pre-existing material in the overlying fields (Riley et al. 2008). This leading edge is identified near the Earth in in-situ observations as the CME sheath region in the solar wind (Forsyth et al. 2006). The darker cavity region is assumed to correspond to a flux rope structure, and is identified as a magnetic cloud (MC; Klein \& Burlaga 1982; Burlaga 1991) in in-situ observations and as a void in HI observations (Howard \& DeForest 2012). The innermost bright feature (CME core) is associated with cold and dense filament material (Webb \& Hundhausen 1987). However, identification of filament material in the in-situ observations near the Earth is rare, and its heliospheric evolution is not well understood (Crooker \& Horbury 2006; Lepri \& Zurbuchen 2010). Also, due to the large distance gap and the difficulty in determining the true evolution of remotely sensed features, association between two sets of observations (remote and in-situ) is still challenging.

The in-situ data taken by the ACE (Stone et al. 1998) and Wind (Ogilvie et al. 1995) spacecraft are analysed to identify the CME structures based on plasma, magnetic field and compositional signatures (Zurbuchen \& Richardson 2006). The in-situ observations from 11 to 12 October 2010 are shown in Figure 9. In this figure, the red curve in the third panel from the top shows the variation of the expected proton temperature described in Lopez (1987) and the first vertical line (dotted, labelled as LE) marks the arrival of the CME leading edge at 05:50 UT on 11 October 2010. The fourth vertical line (dashed, labelled TE) marks the trailing (rear) edge arrival at 17:16 UT. The region bordered by second and third vertical lines (solid), at 09:38 UT and 13:12 UT, respectively, can be classified as a MC (Klein \& Burlaga 1982; Lepping et al. 1990). The enhanced density before the first dotted vertical line is the CME sheath region. Mishra et al. (2014) tracked Feature 1 , the leading edge of the initial density front of the 6 October $2010 \mathrm{CME}$, in $J$-maps and estimated its kinematics based on several methods. The arrival time of Feature 1 is obtained using its kinematics as input to the DBM. Since Feature 1 is the first enhanced density associated with the CME, it is associated with the in-situ measured density enhancement (LE) that arrived at 05:50 UT on 11 October 2010. The time of this density enhancement is within $25 \mathrm{~h}$ of the predicted arrival time of Feature 1 of the CME at L1 (Mishra et al. 2014). 
W. Mishra and N. Srivastava: Heliospheric tracking of density structures of CME

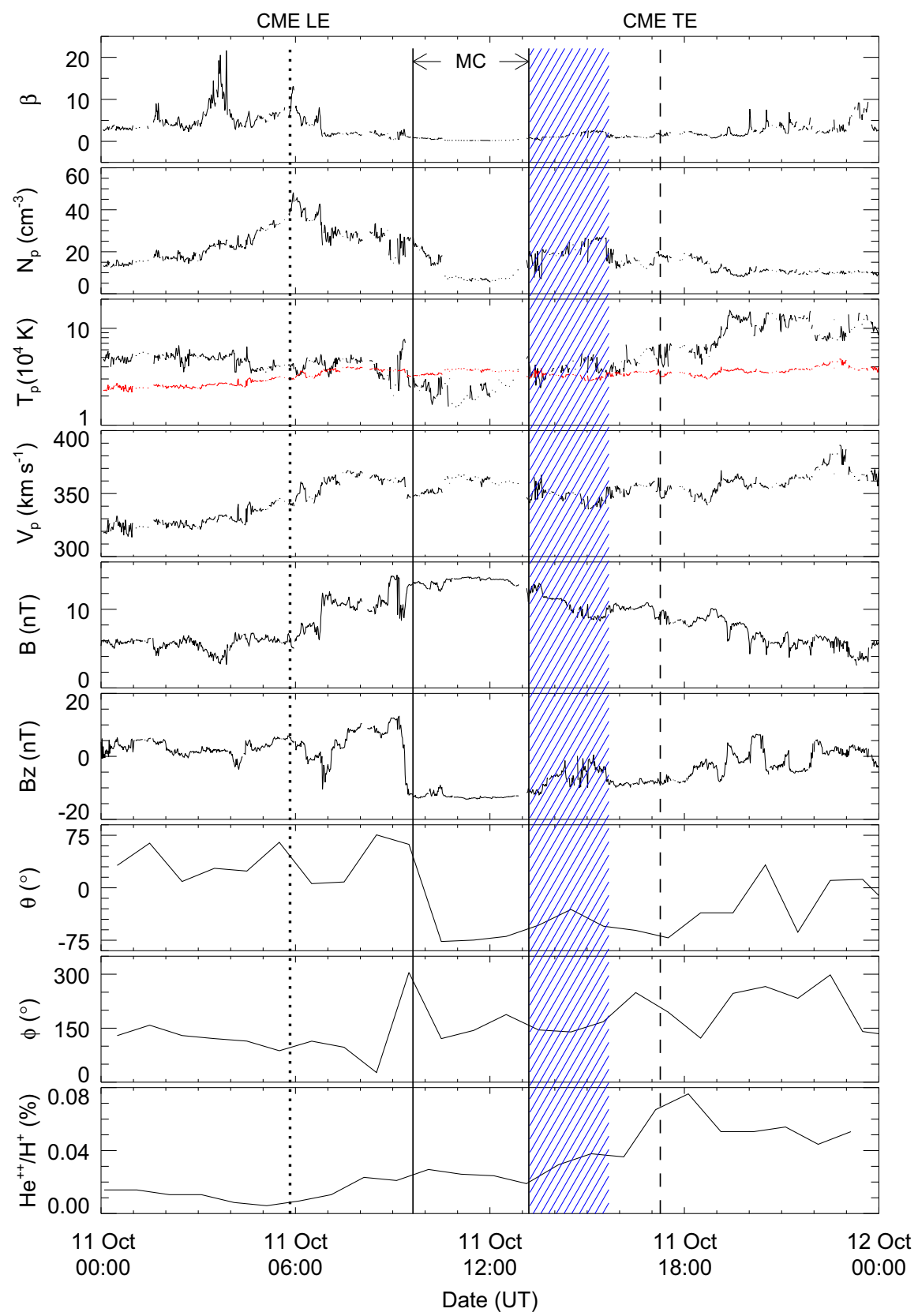

Fig. 9. From top to bottom, panels show plasma beta, proton density, proton temperature, flow speed, magnitude of magnetic field, $z$-component of magnetic field, latitude, longitude of magnetic field vector and alpha to proton ratio. The red curve in the third panel shows the expected proton temperature. From the left, the first (LE), second, third and fourth (TE) vertical lines mark the arrival of CME leading edge, start of magnetic cloud, end of magnetic cloud and trailing edge of CME, respectively. The blue hatched regions mark the region associated with tracked Feature 2.

In Figure 9, we do not notice any monotonic decrease in the speed of the CME, i.e. there is no expansion of MC. This is consistent with the finding that the separation between Feature 1 and Feature 2 became constant well before they reached the L1 point. As discussed in Section 3.2, we expect Feature 2 to arrive at L1, approximately 6-12 h after the arrival of Feature 1. In in-situ observations, we found a second peak in proton density between 13:14 and 15:40 UT on 11 October 2010 and, therefore, associated this with arrival of Feature 2. Feature 2 follows the $\mathrm{MC}$, and is associated with dense (maximum $N_{p}=27 \mathrm{~cm}^{-3}$ ), cold material (minimum $T_{p}=2.4 \times 10^{4} \mathrm{~K}$ ) observed in-situ. It possibly corresponds to the core of a classical three-part structure of a CME.
We need to examine the plasma composition and charge state, and the root mean square deviations of bulk velocity to confirm the presence of filament material in the associated CME at L1 (Burlaga et al. 1998; Gopalswamy et al. 1998; Lepri \& Zurbuchen 2010; Sharma \& Srivastava 2012; Sharma et al. 2013).

Hourly resolution $A C E /$ Solar Wind Ion Composition Spectrometer (SWICS; Gloeckler et al. 1998) data show that the alpha to proton ratio (Fig. 9, lowest panel) is elevated over the entire CME interval, peaking at 0.07 (at 18:30 UT on 11 October 2010) which is seven times the ambient solar wind value (before the leading edge of CME). During the second density enhancement between 13:14 and 15:40 UT on 
11 October 2010, the alpha to proton ratio is approximately three times greater than its value in the ambient solar wind. The $\mathrm{Fe} / \mathrm{O}$ ratio is enhanced up to 0.24 , which is two times larger than its ambient solar wind value, and the $\mathrm{C}^{+6} / \mathrm{C}^{+5}$ ratio reduces to 0.5 and its average value is $\approx 1.0$ thereafter. This enhanced density region corresponds to a decrease in thermal speeds of $\mathrm{He}^{+2}, \mathrm{C}^{+5}, \mathrm{O}^{+7}, \mathrm{Fe}^{+10}$ with minimum values of 18 , 13,12 and $13 \mathrm{~km} \mathrm{~s}^{-1}$, respectively. These signatures are indicative of filament plasma passage during 13:14 to $15: 40$ UT (Burlaga et al. 1998; Gopalswamy et al. 1998; Sharma \& Srivastava 2012). However, during this density enhancement, the $\mathrm{O}^{+7} / \mathrm{O}^{+6}$ ratio, the average charge states of $\mathrm{C}(+5), \mathrm{O}$ $(+6)$ and $\mathrm{Fe}(+10)$ remain roughly constant although they are expected to decrease in a filament region (Lepri \& Zurbuchen 2010). Therefore, although the CME of 6 October 2010 was associated with an eruptive filament, all the criteria required to definitively identify filament plasma are not fulfilled. This may be due to in-situ observations taken at a single point or may occur due to the heating of the cold filament material during its journey to L1 (Skoug et al. 1999; Sharma \& Srivastava 2012).

\section{Results and discussion}

We have attempted to associate two different features of the 6 October $2010 \mathrm{CME}$ in remote and in-situ observations. The two density enhanced features namely Feature 1 and Feature 2 are tracked throughout the combined HI 1 to HI2 field of view, and F1 and F2 are tracked from COR1 to COR2. We applied 10 different reconstruction methods to estimate the 3D kinematics of both features. Interestingly, we found that both features were directed towards the Earth and the separation between them increased with time as they evolved in the heliosphere. Our analysis suggests that both these tracked features are associated with density enhancement at the front and the rear edge of the 6 October 2010 CME. The increasing separation between both features (up to $11.2 R_{\odot}-21.7 R_{\odot}$ ) can be attributed to the expansion of the $\mathrm{CME}$ or unequal forces acting on them.

Based on the in-situ observations, we marked the observed arrival time of features. These marked arrival times at L1 can be compared with the predicted values at L1 obtained from combining remote-sensing kinematics with DBM. The estimated arrival times, transit speeds and errors therein for the Feature 1 are given in Table 1 of Mishra et al. (2014). For the tracked Feature 2, estimated arrival time and transit speed at L1 are given in Table 1 of this paper. Also differences in the estimated values from those measured in-situ are listed in our Table 1. From this table, it is clear that the stereoscopic reconstruction methods which use the simultaneous observation from multiple vantage points give better estimates of the arrival time than those which use observations from single vantage point. This finding is consistent with Mishra et al. (2014) where a comparison of various methods implemented on few CMEs having different characteristics and launched in different solar wind medium was made.

We find that Feature 1 is associated with the density enhancement in the sheath region of the CME while Feature 2 is associated with the density enhancement at the rear edge of a magnetic cloud. The plasma parameters in the second density structure reveal signatures of filament plasma. Association between remotely tracked Feature 2 and second density enhancement measured in-situ is made relying on the kinematics of both features. Since, filaments follow the cavity (flux rope) in imaging (COR) observations, therefore, based on the in-situ measured plasma and compositional data, we expect that the second peak in density corresponding to Feature 2 at the rear edge of magnetic cloud in in-situ measurements is due to arrival of filament structure. Based on our approach of continuous tracking of density structures at the front and rear edge of CME and analysing in-situ data, we express our view that a filament identified in the COR field of view can be tracked further out in the heliosphere using $J$-maps constructed from $\mathrm{HI}$ images. Our detailed examinations of remote and in-situ data reveal that $F 1$ and $F 2$ have been tracked as leading edge and filament in the COR2 field of view as such while Feature 1 and Feature 2 tracked as brightness enhancement (in the HI field of view) could be associated as sheath and filament material of the CME, respectively. We summarize that the features F1 and F2 observed in COR images are related to Feature 1 and Feature 2 in $\mathrm{HI}$ images, respectively.

Although we believe that remotely observed features have been successfully associated with in-situ structures, it is important to remember the fundamental difference between both sets of observations. There is a contribution to the imaging signal from all along the line of sight while the in-situ observations measure density at a particular distance and azimuth in the heliosphere at a time. Moreover, due to a fixed single point location of in-situ spacecraft, it is difficult to claim that remotely tracked features are certainly intersected by the in-situ spacecraft unless we track them up to L1 which could not be done in the present case. From the present study, we also realize that the use of difference images for tracking of cavity (observed as magnetic cloud in in-situ) which is expected to lie between the two tracked features may be difficult. It is because; density associated with cavity (flux rope) becomes indistinguishable from the brighter background on taking the running difference. However, from our study limited to a single CME, tracking of filaments seems to be possible using running difference images of SECCHI/COR and HI.

Acknowledgements. The authors thank T.A. Howard for his valuable comments and useful discussions. We also thank the referees and editor whose comments and suggestions have helped to improve the manuscript considerably. We thank the team members of STEREO, ACE and Wind spacecraft for the data used in this paper. The in-situ measurements of solar wind were obtained from NASA CDAWeb (http://cdaweb.gsfc.nasa.gov). We acknowledge the UK Solar System Data Centre for providing the processed Level 2 STEREO/HI data. The editor thanks David F. Webb and an anonymous referee for their assistance in evaluating this paper.

\section{References}

Billings, D.E. A guide to the solar corona. Academic Press, New York, 150, 1966.

Burlaga, L., R. Fitzenreiter, R. Lepping, K. Ogilvie, A. Szabo, et al. A magnetic cloud containing prominence material - January 1997. J. Geophys. Res., 103, 277, 1998.

Burlaga, L.F.E. Magnetic Clouds. In: R. Schwenn, and E. Marsch, Editors, Physics of the Inner Heliosphere II, Springer, Berlin, $1-2,1991$

Cargill, P.J. On the aerodynamic drag force acting on interplanetary coronal mass ejections. Sol. Phys., 221, 135-149, 2004.

Cargill, P.J., J. Chen, D.S. Spicer, and S.T. Zalesak. Magnetohydrodynamic simulations of the motion of magnetic flux tubes through a magnetized plasma. J. Geophys. Res., 101, 4855-4870, 1996.

Colaninno, R.C., A. Vourlidas, and C.C. Wu. Quantitative comparison of methods for predicting the arrival of coronal mass ejections at Earth based on multiview imaging. J. Geophys. Res., 118, 6866-6879, 2013. 
Crooker, N.U., and T.S. Horbury. Solar imprint on ICMEs, their magnetic connectivity, and heliospheric evolution. Space Sci. Rev., 123, 93-109, 2006.

Davies, J.A., R.A. Harrison, A.P. Rouillard, N.R. Sheeley, C.H. Perry, et al. A synoptic view of solar transient evolution in the inner heliosphere using the Heliospheric Imagers on STEREO. Geophys. Res. Lett., 36, L02102, 2009.

Davies, J.A., R.A. Harrison, C.H. Perry, C. Möstl, N. Lugaz, et al. A self-similar expansion model for use in solar wind transient propagation studies. Astrophys. J., 750, 23, 2012.

Davies, J.A., C.H. Perry, R.M.G.M. Trines, R.A. Harrison, N. Lugaz, C. Möstl, Y.D. Liu, and K. Steed. Establishing a stereoscopic technique for determining the kinematic properties of solar wind transients based on a generalised self-similarly expanding circular geometry. Astrophys. J., 777, 167, 2013.

Davis, C.J., J.A. Davies, M. Lockwood, A.P. Rouillard, C.J. Eyles, and R.A. Harrison. Stereoscopic imaging of an Earth-impacting solar coronal mass ejection: a major milestone for the STEREO mission. Geophys. Res. Lett., 36, L08102, 2009.

Davis, C.J., J. Kennedy, and J.A. Davies. Assessing the accuracy of CME Speed and trajectory estimates from STEREO observations through a comparison of independent methods. Sol. Phys., 263, 209-222, 2010.

DeForest, C.E., T.A. Howard, and S.J. Tappin. Observations of detailed structure in the solar wind at $1 \mathrm{AU}$ with STEREO/HI-2. Astrophys. J., 738, 103, 2011

Eyles, C.J., R.A. Harrison, C.J. Davis, N.R. Waltham, B.M. Shaughnessy, et al. The heliospheric imagers onboard the STEREO mission. Sol. Phys., 254, 387-445, 2009.

Forsyth, R.J., V. Bothmer, C. Cid, N.U. Crooker, T.S. Horbury, et al. ICMEs in the inner heliosphere: origin, evolution and propagation effects. report of working group G. Space Sci. Rev., 123, 383-416, 2006.

Gloeckler, G., J. Cain, F.M. Ipavich, E.O. Tums, P. Bedini, et al. Investigation of the composition of solar and interstellar matter using solar wind and pickup ion measurements with SWICS and SWIMS on the ACE spacecraft. Space Sci. Rev., 86, 497-539, 1998.

Gopalswamy, N., Y. Hanaoka, T. Kosugi, R.P. Lepping, J.T. Steinberg, et al. On the relationship between coronal mass ejections and magnetic clouds. Geophys. Res. Lett., 25, 2485-2488, 1998.

Gopalswamy, N., A. Dal Lago, S. Yashiro, and S. Akiyama. The expansion and radial speeds of coronal mass ejections. Central European Astrophysical Bulletin, 33, 115-124, 2009.

Harrison, R.A., J.A. Davies, C. Möstl, Y. Liu, M. Temmer, et al. An analysis of the origin and propagation of the multiple coronal mass ejections of 2010 August 1. Astrophys. J., 750, 45, 2012.

Howard, R.A., J.D. Moses, A. Vourlidas, J.S. Newmark, D.G. Socker, et al. Sun Earth Connection Coronal and Heliospheric Investigation (SECCHI). Space Sci. Rev., 136, 67-115, 2008.

Howard, T.A. Three-dimensional reconstruction of coronal mass ejections using heliospheric imager data. J. Atmos. Sol. Terr. Phys., 73, 1242-1253, 2011.

Howard, T.A., and C.E. DeForest. Inner heliospheric flux rope evolution via imaging of coronal mass ejections. Astrophys. J., 746, 64, 2012

Howard, T.A., and S.J. Tappin. Interplanetary coronal mass ejections observed in the heliosphere: 1. review of theory. Space Sci. Rev., 147, 31-54, 2009.

Howard, T.A., D.F. Webb, S.J. Tappin, D.R. Mizuno, and J.C. Johnston. Tracking halo coronal mass ejections from $0-1 \mathrm{AU}$ and space weather forecasting using the solar mass ejection imager (SMEI). J. Geophys. Res., 111, A04105, 2006.

Illing, R.M.E., and A.J. Hundhausen. Observation of a coronal transient from 1.2 to 6 solar radii. J. Geophys. Res., 90, 275-282, 1985.

Joshi, A.D., and N. Srivastava. Kinematics of two eruptive prominences observed by EUVI/STEREO. Astrophys. J., 730, $104,2011$.
Kahler, S.W., and D.F. Webb. V arc interplanetary coronal mass ejections observed with the solar mass ejection imager. J. Geophys. Res., 112, A09103, 2007.

Klein, L.W., and L.F. Burlaga. Interplanetary magnetic clouds at 1 AU. J. Geophys. Res., 87, 613-624, 1982.

Lemen, J.R., A.M. Title, D.J. Akin, P.F. Boerner, C. Chou, et al. The atmospheric imaging assembly (AIA) on the solar dynamics observatory (SDO). Sol. Phys., 275, 17-40, 2012.

Lepping, R.P., L.F. Burlaga, and J.A. Jones. Magnetic field structure of interplanetary magnetic clouds at 1 AU. J. Geophys. Res., 95, 11957-11965, 1990.

Lepri, S.T., and T.H. Zurbuchen. Direct observational evidence of filament material within interplanetary coronal mass ejections. Astrophys. J. Lett., 723, L22-L27, 2010.

Liu, Y., J.A. Davies, J.G. Luhmann, A. Vourlidas, S.D. Bale, and R.P. Lin. Geometric triangulation of imaging observations to track coronal mass ejections continuously out to 1 AU. Astrophys. J. Lett., 710, L82-L87, 2010a.

Liu, Y., A. Thernisien, J.G. Luhmann, A. Vourlidas, J.A. Davies, R.P. Lin, and S.D. Bale. Reconstructing coronal mass ejections with coordinated imaging and in situ observations: global structure, kinematics, and implications for space weather forecasting. Astrophys. J., 722, 1762-1777, 2010b.

Liu, Y., J.G. Luhmann, S.D. Bale, and R.P. Lin. Solar source and heliospheric consequences of the 2010 April 3 coronal mass ejection: a comprehensive view. Astrophys. J., 734, 84, 2011.

Liu, Y.D., J.G. Luhmann, N. Lugaz, C. Möstl, J.A. Davies, S.D. Bale, and R.P. Lin. On sun-to-earth propagation of coronal mass ejections. Astrophys. J., 769, 45, 2013.

Lopez, R.E. Solar cycle invariance in solar wind proton temperature relationships. J. Geophys. Res., 92, 11189-11194, 1987.

Lugaz, N. Accuracy and limitations of fitting and stereoscopic methods to determine the direction of coronal mass ejections from heliospheric imagers observations. Sol. Phys., 267, 411-429, 2010

Lugaz, N., A. Vourlidas, and I.I. Roussev. Deriving the radial distances of wide coronal mass ejections from elongation measurements in the heliosphere - application to CME-CME interaction. Ann. Geophys., 27, 3479-3488, 2009.

Lugaz, N., J.N. Hernandez-Charpak, I.I. Roussev, C.J. Davis, A. Vourlidas, and J.A. Davies. Determining the azimuthal properties of coronal mass ejections from multi-spacecraft remote-sensing observations with STEREO SECCHI. Astrophys. J., 715, 493-499, 2010.

Manoharan, P.K. Evolution of coronal mass ejections in the inner heliosphere: a study using white-light and scintillation images. Sol. Phys., 235, 345-368, 2006.

Michalek, G., N. Gopalswamy, and S. Yashiro. Expansion speed of coronal mass ejections. Sol. Phys., 260, 401-406, 2009.

Mierla, M., B. Inhester, C. Marqué, L. Rodriguez, S. Gissot, A.N. Zhukov, D. Berghmans, and J. Davila. On 3D reconstruction of coronal mass ejections: I. method description and application to SECCHI-COR data. Sol. Phys., 259, 123-141, 2009.

Mishra, W., and N. Srivastava. Estimating the arrival time of earthdirected coronal mass ejections at in situ spacecraft using COR and HI observations from STEREO. Astrophys. J., 772, 70, 2013.

Mishra, W., and N. Srivastava. Morphological and kinematic evolution of three interacting coronal mass ejections of 2011 February 13-15. Astrophys. J., 794, 64, 2014.

Mishra, W., N. Srivastava, and J.A. Davies. A comparison of reconstruction methods for the estimation of coronal mass ejections kinematics based on SECCHI/HI observations. Astrophys. J., 784, 135, 2014.

Mishra, W., N. Srivastava, and D. Chakrabarty. Evolution and consequences of interacting CMEs of 9-10 November 2012 using STEREO/SECCHI and in situ observations. Sol. Phys., 290, 527-552, 2015

Möstl, C., and J.A. Davies. Speeds and arrival times of solar transients approximated by self-similar expanding circular fronts. Sol. Phys., 285, 411-423, 2013. 
Möstl, C., T. Rollett, N. Lugaz, C.J. Farrugia, J.A. Davies, et al. Arrival time calculation for interplanetary coronal mass ejections with circular fronts and application to STEREO observations of the 2009 February 13 eruption. Astrophys. J., 741, 34, 2011.

Ogilvie, K.W., D.J. Chornay, R.J. Fritzenreiter, F. Hunsaker, J. Keller, et al. SWE, a comprehensive plasma instrument for the Wind spacecraft. Space Sci. Rev., 71, 55-77, 1995.

Poomvises, W., J. Zhang, and O. Olmedo. Coronal mass ejection propagation and expansion in three-dimensional space in the heliosphere based on Stereo/SECCHI observations. Astrophys. J. Lett., 717, L159-L163, 2010.

Riley, P., R. Lionello, Z. Mikić, and J. Linker. Using global simulations to relate the three-part structure of coronal mass ejections to in situ signatures. Astrophys. J., 672, 1221-1227, 2008.

Rouillard, A.P., J.A. Davies, R.J. Forsyth, A. Rees, C.J. Davis, et al. First imaging of corotating interaction regions using the STEREO spacecraft. Geophys. Res. Lett., 35, L1011, 2008.

Schwenn, R., A. dal Lago, E. Huttunen, and W.D. Gonzalez. The association of coronal mass ejections with their effects near the Earth. Ann. Geophys., 23, 1033-1059, 2005.

Sharma, R., and N. Srivastava. Presence of solar filament plasma detected in interplanetary coronal mass ejections by in situ spacecraft. J. Space Weather Space Clim., 2 (26), A10, 2012.

Sharma, R., N. Srivastava, D. Chakrabarty, C. Möstl, and Q. Hu. Interplanetary and geomagnetic consequences of 5 January 2005 CMEs associated with eruptive filaments. J. Geophys. Res., 118, 3954-3967, 2013.
Sheeley, N.R., J.H. Walters, Y.-M. Wang, and R.A. Howard. Continuous tracking of coronal outflows: two kinds of coronal mass ejections. J. Geophys. Res., 104, 24739-24768, 1999.

Sheeley Jr., N.R., A.D. Herbst, C.A. Palatchi, Y.-M. Wang, R.A. Howard, et al. Heliospheric images of the solar wind at Earth. Astrophys. J., 675, 853-862, 2008.

Skoug, R.M., S.J. Bame, W.C. Feldman, J.T. Gosling, D.J. McComas, et al. A prolonged $\mathrm{He}^{+}$enhancement within a coronal mass ejection in the solar wind. Geophys. Res. Lett., 26, 161-164, 1999.

Stone, E.C., A.M. Frandsen, R.A. Mewaldt, E.R. Christian, D. Margolies, J.F. Ormes, and F. Snow. The advanced composition explorer. Space Sci. Rev., 86, 1-22, 1998.

Tappin, S.J., A. Buffington, M.P. Cooke, C.J. Eyles, P.P. Hick, et al. Tracking a major interplanetary disturbance with SMEI. Geophys. Res. Lett., 31, L02802, 2004.

Thompson, W.T. 3D triangulation of a Sun-grazing comet. Icarus, 200, 351-357, 2009.

Vršnak, B., T. Žic, T.V. Falkenberg, C. Möstl, S. Vennerstrom, and D. Vrbanec. The role of aerodynamic drag in propagation of interplanetary coronal mass ejections. $A \& A, \mathbf{5 1 2}, \mathrm{A} 43,2010$.

Vršnak, B., T. Žic, D. Vrbanec, M. Temmer, T. Rollett, et al. Propagation of interplanetary coronal mass ejections: the dragbased model. Sol. Phys., 285, 295-315, 2013.

Webb, D.F., and A.J. Hundhausen. Activity associated with the solar origin of coronal mass ejections. Sol. Phys., 108, 383-401, 1987.

Zurbuchen, T.H., and I.G. Richardson. In-situ solar wind and magnetic field signatures of interplanetary coronal mass ejections. Space Sci. Rev., 123, 31-43, 2006.

Cite this article as: Mishra W \& Srivastava N. Heliospheric tracking of enhanced density structures of the 6 October 2010 CME. J. Space Weather Space Clim., 5, A20, 2015, DOI: 10.1051/swsc/2015021. 